\title{
LEARNING FROM THE LIVING BODIES: NEW PRINCIPLES AND TECHNIQUES FOR PHOTONIC SENSING
}

\author{
José A. Martín-Pereda ${ }^{a}$ \\ Universidad Politécnica de Madrid \\ Ciudad Universitaria. 28040 Madrid. Spain
}

\begin{abstract}
Signal processing in any living being is much more complex than the one performed in artificial systems. Cortex architecture, although only partly known, gives some useful ideas to be employed in sensing technology. To analyze some of these structures is the objective of this paper. Among the points to be analyzed are the parallel transfer of information, the similarity of the different systems and the massive amount of data analyzed by physical techniques. As an example of these concepts, the possibility to transmit images in a parallel way will be reported.
\end{abstract}

Keywords: Photonic sensing, visual cortex, parallel processing.

\section{INTRODUCTION}

All animals have to deal with their world and the objects in it. They walk on objects, crawl under them, avoid crashing into them, pick them up, eat them, mate with them, run away from them. Back in the biological dawn, when evolution was younger, much younger than today, animals had to make physical contact with objects before they could tell that those objects were there. A bonanza of benefit was given to them when they developed a remote-sensing technology. They were aware of an obstacle before hitting it, or a predator before being seized, or food being anywhere in the large vicinity. This result came from two main sources, the sun and the evolution of those magical devices we call eyes. Many authors say that eyes have evolved no fewer than forty times; some others give larger numbers, sixty or, perhaps, seventy. They have evolved independently in various parts of the animal kingdom and, in some cases, they use radically different principles. Nine distinct ideas have been recognized among those fifty or seventy independently evolved eyes. And each one of them is present according to the circumstantial need. Similar reasoning may be indicated with respect to any other sensing capability of the living bodies. Billions of years have been dedicated to this evolution. Hundreds of trial and error steps have appeared and disappeared along this time. Results are around us and, in some cases, it is impossible to imagine better sensing instruments.

The main question in this paper is, can the present information we have about biological sensing and the involved principles be exploited to develop sensing devices that can be applied to technology that will provide for human needs? This paper will not solve this question but will try to offer some ideas concerning some of those solutions given by Nature to its living beings. Following lines will underline a few selection of basic facts extracted from fundamentals of Sensory Physiology and will offer simple examples of how these facts may be implemented. More than offer results, this paper will present initial concepts.

Another point needs to be considered. The principles underlying all sensory perceptions are similar. As it will be shown, different sense organs very much resemble one another, both in their organization and operation and in their connections to the central nervous system. Most of the author' work has been related with the visual system and it is because that this sense will be the principal subject of this paper. But similar principles are present in other sensing systems and these analogies will be indicated. A last factor arises. Dealing with living beings sensory perception one encounters the problem of subjectivity. That is, environmental stimuli and the respective responses of their sense organs correspond to statements by the subject about his sensations and perceptions. The high-level mental aspects of sensory physiology appear far richer than the obtained from plain low-level sense organs. A living being is nothing other than the sum of his experiences. This should be the final step in the system and will deserve some comments of this paper.

\footnotetext{
a jamp@tfo.upm.es
} 


\section{SOME BASIC CONCEPTS IN SENSORY PHYSIOLOGY}

Althought external stimuli have very different roots and they act on different senses, internal processing is always similar. Once the stimulus has been received by the sensing element, the characteristics of the neural path, from receptor to cortex, follow similar rules and patterns. It is because that a common scheme can be given for all sensing biological systems.
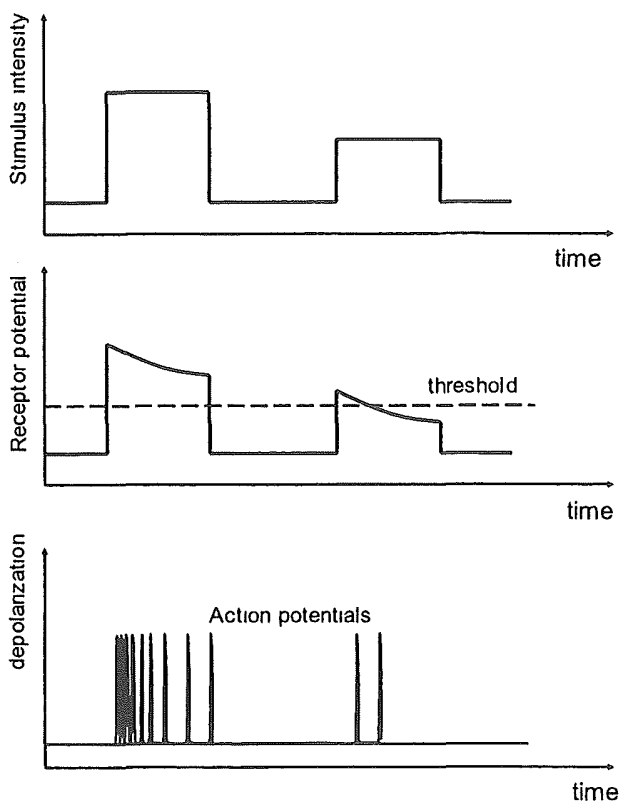

Fig. 1.- Relationships between stimulus intensity and frequency of action potentials. This first part of the paper will ennumerate some general facts in every sensory system as well as the characteristics of neuronal responses to external stimuli and how these neurons are arranged. This part will serve as tutorial for these concepts and will give the fundamental ideas to the present goal. Some more details may be seen in ${ }^{1-5}$.

\subsection{Initiation of Train of Impulses}

Living bodies experience their environment not directly, and not in their entirety, but rather by way of specialized sense organs. Environmental influences give to it the corresponding information and that information goes to the central nervous system (CNS). Whereas sensory impression is given by its modality - sight, hearing, touch, taste and smell - and quality for example, red, green, blue - its intensity is given by its quantity. The quantity of a sensory impression corresponds to the strength of the stimulus. The general nature of the response of a receptor to stimuli of increasing intensity appears in Fig. 1. The receptor potential becomes larger and the frequency of the triggered action potentials increases.

\subsection{Microcircuits}

Excitation and inhibition by single synapses usually have little behavioral significance by themselves; it is the assembly of synapses into patterns of connectivity during development that produces functionally significant operations. The basic types are synaptic divergence and convergence and inhibitory.

Synaptic divergence provides divergence of output from a single terminal (fan-out). In fig. 2.A a presynaptic terminal (a) has excitatory synapses into postsynaptic dendrites (b-f). An action potential (ap) invading the presynaptic

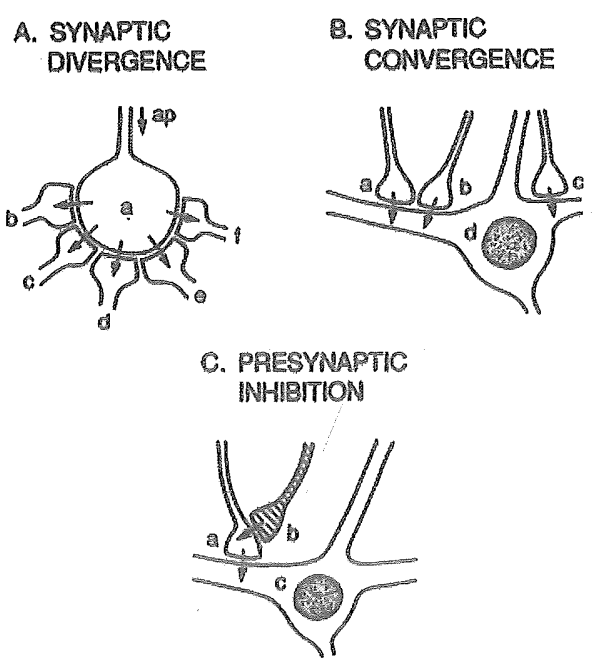

Fig. 2.- The simplest types of microcircuits. A. Synaptic divergence. B. Sinaptic convergence. C. Presynaptic inhibition. terminal can thus cause simultaneous excitatory postsynaptic potentials (EPSPs) in many postsynaptic dendrites. The advantage of this arrangement is that activity in a single axon is amplified into activity in many postsynaptic neurons, conferring a high gain upon the system. The activity is simultaneous, thus retaining the timing of the input, and it is of the same sign: excitation gives excitation.

The considerable divergence that characterizes the output of a neuron in matched by convergence of many inputs (fan-in). In Fig. 2.B, two terminals (a and $\mathbf{b}$ ) make synapses onto a postsynaptic dendrite (d). If both terminals are excitatory, spread of an impulse into terminal (a) sets up an EPSP; slightly later, spreads of an impulse into terminal (b) set up an EPSP which summates with that of (a). This is named temporal summation. Although impulses in (a) and (b) may be asynchronous, their EPSPs nonetheless can summate. This is a nonlinear process. Synaptic convergence also involves summation of excitatory and inhibitory PSPs. In addition to temporal summation, there is spatial summation of responses arising in different parts of a dendrite, as well as different parts of the whole dendritic tree. Spatial summation allows for combining of many inputs into one integrated postsynaptic response. The separation of PSPs reduces some of the nonlinear interaction between synaptic conductances, making the 
summation more linear.

Presynaptic inhibition is the final type of simple synaptic combination involving convergence. In this arrangement (Fig. 2.C), a prsynaptic terminal (a) is itself postsypnaptic to another terminal (b). The presynaptic action

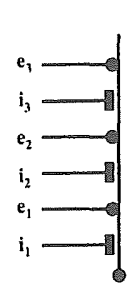

(A)
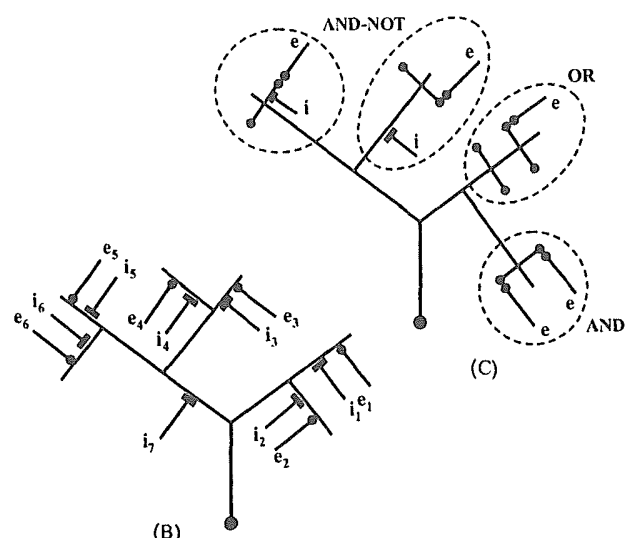

(C)

(B)

Fig. 3.- Possible arrangements of synapses that have the potential for subserving logic operations. (A) A single dendrite receives excitatory (e) and inhibitory (i) synapses. (B) Branching dendritic tree with e and I synapses. (C) Branching dendritic tree with e svnapses on spines. may involve a conventional type of IPSP produced by (b) in the presynaptic terminal (a). Alternatively, there may be a maintained depolarization of the presynaptic terminal, reducing the amplitude of an invading impulse and with it the amount of transmitter released from the terminal. The essential operating characteristic of this microcircuit is that the effect of an input (a) on cell (c) can be reduced or abolished (by b) without there being any direct action of (b) on the cell (c) itself. Control of the input (a) to the dendrite or cell body can thus be much more specific.

\subsection{Dendritic computation}

The importance of the sites and types of synaptic inputs on a dendritic branch can be illustrated by using the paradigm of logic operations. In the diagram of Fig. 3.A, alternating excitatory and inhibitory synapses are arranged along a dendritic branch. Given the nonlinear interaction between these synapses, an inhibitory synapse $(\mathrm{i} 1, \mathrm{i} 2, \mathrm{i} 3)$ with a synaptic reversal potential close to the resting potential of the cell can effectively oppose the ability of a membrane potential change generated by any more distal excitatory synapse to spread to the soma and generate impulses there. By contrast, an inhibitory synapse has little effect in vetoing the voltage change initiated by more proximal excitatory synapses. This operation is an analog form of a digital AND-NOT gate (e and not more proximal i) and is a mechanism underlying various computations, such as direction selectivity in retinal ganglion cells.

This type of synaptic arrangement can also be found in more localized parts of a dendritic tree. Fig. 3.B depicts a case in which a dendritic has numerous distal branches, each with excitatory and an inhibitory synapse. The same "on-path" role still applies. Finally, Fig. 3.C represents a dendritic tree with its distal branches covered by spines. One possible arrangement is that the impulse would fire if any one of several spines in a cluster should receive an excitatory input; this would be equivalent to an OR gate. Alternatively, two simultaneous inputs might be required; this would constitute an AND gate. Finally, one might have AND-NOT gates. Depending on the placement of the inhibition, the gate might be localized to an individual spine, or might involve a dendritic branch containing a cluster of spines.

\subsection{Basic Circuit for Cortical Organization}

The main and striking fact is that the local circuit elements are common to the different types of cortex. Inhibitory circuits play large roles in determining the types of operations generated within a region. Three are the principal architectures.

Rhythmic generation. A common model of this activity appears in Fig. 3.A. It consists of output neurons (b) connected through axon collaterals to inhibitory neurons (i), which in turn connect back into the output neurons. When an input (a) activates the output neurons, they begin to generate impulses, which lead to activation of interneurons. This activation leads to feedback inhibition of the output neurons, which now can no longer respond to the input and thus also deprives the interneurons of their source of activation; they are, in a sense, presynaptically inhibited by themselves. Both populations are silent until the IPSPs in the output neurons wear off and the cycle is ready to be repeated. The degree of shynchronization of a region depends on the extensiveness of the connections. Evidence in both olfactory bul and olfactory cortex as well as in the visual cortex relates these oscillations to perception and behavior.

Receptive field organization. Inhibitory circuits play an important role in spatial contrast, a common property of receptive field organization in many sensory systems. This property is illustrated in Fig. 4.B, where there is strong stimulation of input $\left(a_{1}\right)$ and weaker stimulation of input $\left(a_{2}\right)$. The responses of $b_{1}$ and $b_{2}$ would start out being proportional; however, the stronger inhibition by $b_{1}$ and $i_{1}$ suppresses $b_{2}$ more than the suppression of $b_{1}$ by $b_{2}$ and $i_{2}$, 


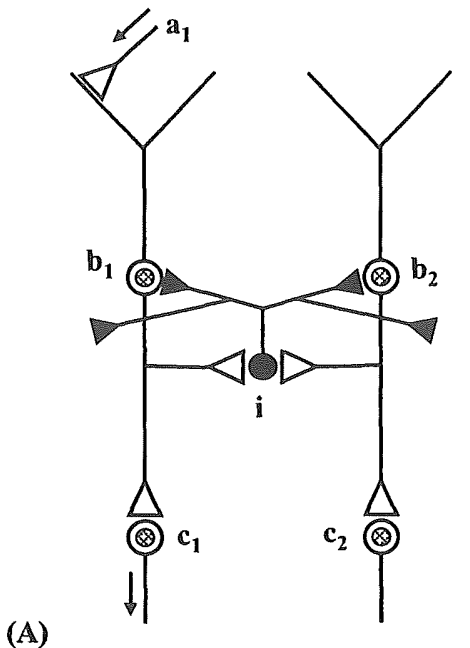

(A)

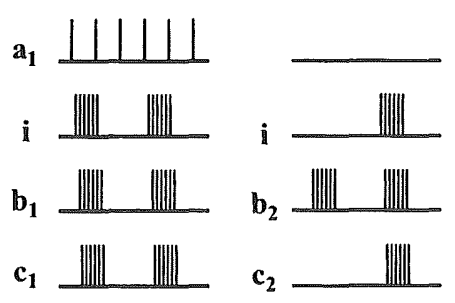

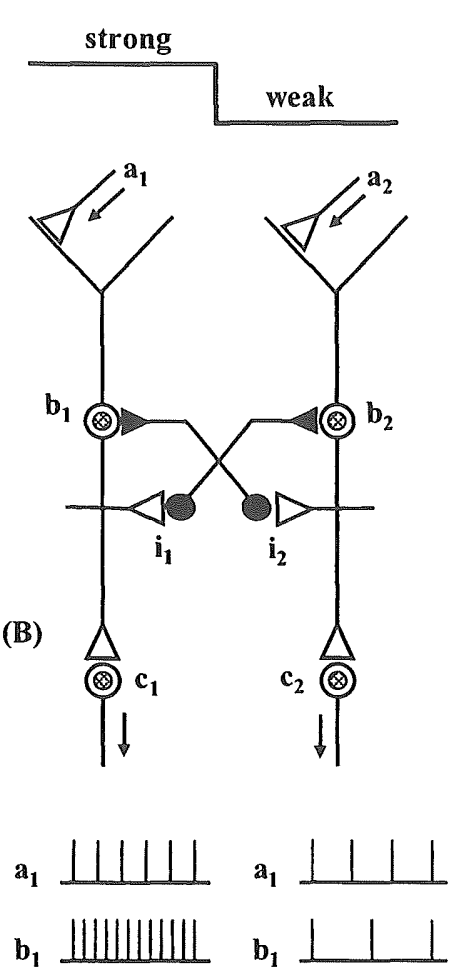

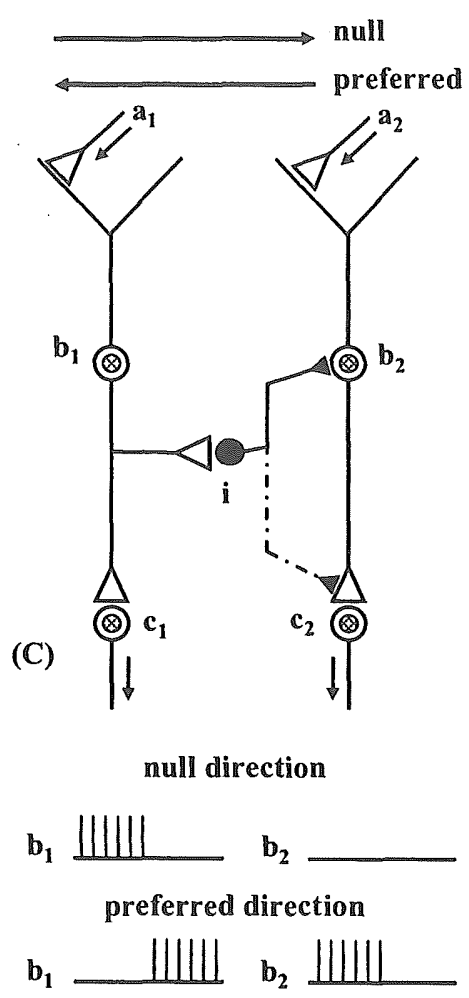

Fig. 4.- Intrinsic inhibitory circuits. (A) Rhythm generation. Impulse firing patterns are shown below. (B) Spatial contrast with strong and weak areas of stimulation. (C) Direction selectivity.

thereby enhancing the difference in firing rates of $b_{1}$ and $b_{2}$. This is the basis for the classical description of Mach bands in the visual system.

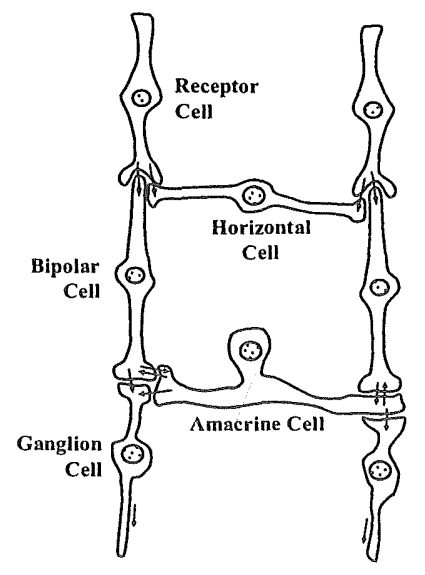

(A)

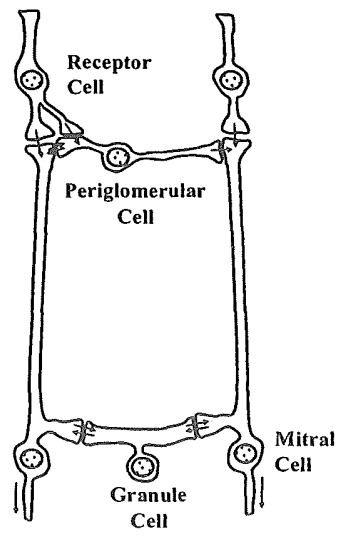

(B)

Fig. 5.- Comparison between basic circuits for the retina (A) and olfactory bulb (B) to perform most of the operations appearing in some region of the cortex. In Fig. 5 is shown a comparison between basic

Directional selectivity. A third type with an inhibitory interneuron behavior plays an essential role in direction selectivity. The best elaborated model is the retina. The model is shown in Fig. 4.C. The essential element is an inhibitory interneuron whose connections are made in one direction, opposite to the preferred direction. This means that stimuli moving in that direction, called the "null" direction, activate the inhibitory connections in that direction, so that cells further along cannot respond. In the opposite, preferred direction, by contrast, the cells are free to respond.

From above concepts, it is possible to define a basic circuit as a flexible tool, not rigidly defined, able 
circuits for the retina (A) and olfactory bulb (B). Despite the fact that these regions process entirely different sensory modalities, the basic circuits are similar in outline and in several details. In each case, there are parallel vertical pathways for straight-through transmission of information. In addition, there are horizontal connections, arranged in two main levels, for processing of information by lateral interactions. Within this framework are further similarities, such as reciprocal synapses and interneurons that lack axons.

Among the common principles in these two circuits are: there is an initial stage of input processing, a second stage of intrinsic operations within the synaptic circuits of the region, and a final stage of output control.

\subsection{From Synaptic Circuits to Network Properties}

From previous circuits a higher level appears with millions of synapses. These networks show emergent behaviors that may not be apparent or implied in their constituent elements. Examples of these emergent properties are shown in Fig. 6. Here are represented three connection schemes possibly underlying orientation selectivity in the mammalian visual cortex. Whereas cells in the lateral geniculate nucleus have circular symmetrical receptive fields, their targets in primary visual cortex have elongated receptive fields, responding best to an oriented bar (Fig. 6.A). A large number of different wiring schemes have been proposed to explain this phenomenon, being the main one that a number of geniculate cells, with their receptive field centers arranged in a row, converge onto cortical cell (Fig. 6.B). Another class of models involves the use of the feedforward type of inhibition to prevent the cortical cell from firing if the visual stimulus falls on

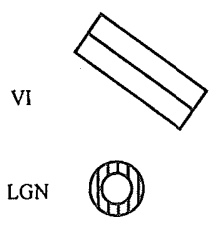

(A)
(B)

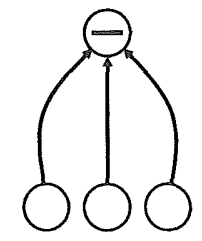

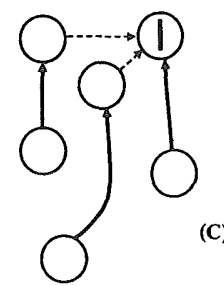

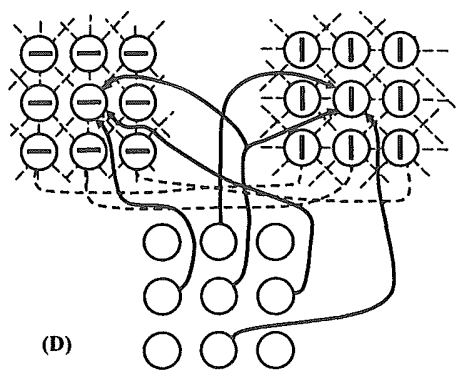
a neighboring region, outside the bar-shaped receptive field (Fig. 6.C; "iso-orientation inhibition"). The same operation can be accomplished by using a massively interconnected network of inhibitory cells, as indicated in Fig. 6.D. If a vertical bar stimulus falls on the receptive fields of the central row of the three geniculate cells, cortical cells in the vertical orientation column (right side in Fig. 6.D) will be optimally activated and the cell will fire. These cortical neurons also inhibit cells (dashed lines) in the horizontal orientation column (left side in Fig. 6.D). This sort of inhibition is referred to as cross-orientation inhibition. If the bar is now tilted by $45^{\circ}$, such as to excite the diagonally located geniculate cells, local inhibition among similarly oriented cortical neurons (dotted lines) will prevent, or at least reduce, the excitability of the cortical cells in the vertical orientation column. Similarly, if the stimulus bar is vertical but brad, covering more than the central row of geniculate cells, the cortical response will be muted due to this

Fig. 6.- Some examples of the way by which a selective response arises as a network nronertv.

instance of lateral inhibition. An important consequence is that no single neuron is solely responsible for this orientation tuning, since they act synergistically.

\subsection{Convergence and Divergence}

Once the superficial sensing units have received the external signal, a first processing step y carried out in a few cell layers. Usually, the number of sensing cells, in the first stratum, is much larger than the corresponding to neurons at successive layers. This situation allows the complex behavior indicated in previous paragraph, where some cells networks are able to detect complex images. One rod diverges at successive stages to two, five, and finally eight interneurons. Thus, the signal arising in one rod would expand to eight copies at the level of the bipolar axon, and this would mobilize several hundred synapses onto beta ganglion cells. This would amplify the signal and thus protect it from certain corruption by noise at the level of the ganglion cell spike generator. The reconvergence occurring at the last stage of this circuit (from eight copies in the bipolar to two in the on-beta cell) tend to remove accumulated synaptic noise by signal averaging: The eight copies of the signal are time-locked and thus mutual reinforcing when they converge. 


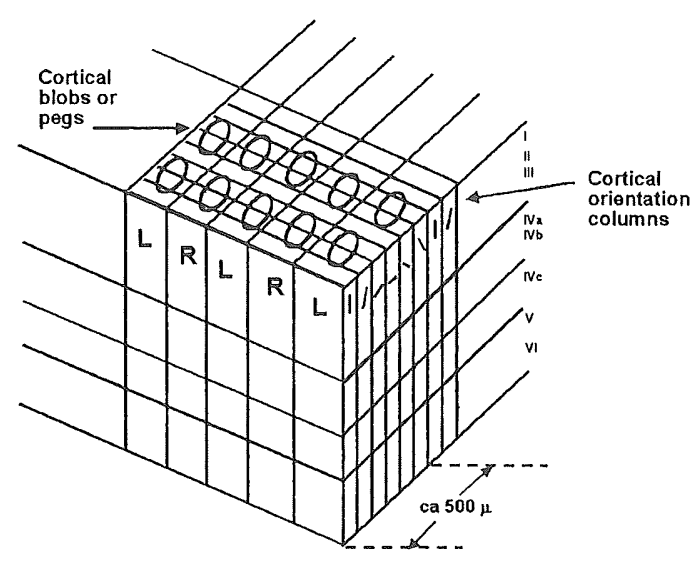

Fig. 7.- Structure of a part of the mammalian visual cortex.
However, the synaptic noise accompanying each copy of the signal is temporally random and thus not reinforced by the convergence. At the last stage in this circuit there is divergence of the signal to at least two on-beta cells.

\subsection{Cortex structure}

The information perceived in the sensing level goes now to its final destination in the cortex. Each zone of it is devoted to a particular type of sense. The area related with the visual cortex will be the object of this paragraph.

The whole structure and function of the neuron primary visual cortex architecture is somehow much more complex than previous structures. Their nerve cells are arranged in six distinct cytoarchitectonic layers parallel to the cortical surface. These nerve cells are organized not only within layers parallel to the surface but also in "columns" perpendicular to it. When the neurons laying at these columns are tested with contours oriented in various

directions one finds a progressive change in optimal orientation across adjacent columns. In other words, the cells in a particular column share the same preferred orientation. This orientation changes in a systematic way as one move across the cortical surface, such that after half a millimeter or so we are back to the first orientation. Thus the visual cortex is traversed by a series of bands, within each of which every possible orientation is represented. This fact may be demonstrated anatomically. A highly stylized representation of a slab of visual cortex, showing its organization into columns, is shown in Fig. 7. Right and left eyes are at alternate columns. It is necessary to travel a minimum distance of about $0.8 \mathrm{~mm}$ for the cells to respond to the right eye plus the left eye and then start the sequence again, a distance comprising the orientation hypercolumn. Equally, it is necessary to travel about $1 \mathrm{~mm}$ for all 18 orientations to be covered, a distance comprising the orientation hypercolumn. Each small part of the field of view is screened for one eye and then for the other eye, and each small part is simultaneously screened for different orientations, the entire process being repeated again in the adjacent millimeter for an adjacent small part of the field of view. Colors are detected, in a similar way, at specific places, named cortical blobs or pegs.

\subsection{Synthesis of main factors to be considered}

Although many other factors should be considered, in order to summarize some of the vertebrate nervous system characteristics, we present now some of the main factors to considerer in present work. These points, in a much synthesized form are the following:

a. Different aspects of the visual scene, as seen by living beings, are perceived in a parallel way.

b. Each portion of the visual scene goes through the different layers of the retina by different paths.

c. Information about the relation between the information obtained from a particular area and information from adjacent areas is transferred to following layers, by a particular frequency coding in adjacent neuronal paths.

d. Information about the intensity of each one of the scene details are transferred from the third retina layer to following levels after a conversion from intensity level to frequency. Lower intensities correspond with lower frequencies.

e. Information about different line orientations is transferred to selected areas at the visual cortex. These areas become excited by this information and living beings get a stimulus concerning that orientation in the visual scene.

f. Each particular orientation, or each particular shape, goes to a precise area in the V1 area of the visual cortex.

g. Information is always transferred in a parallel way.

h. Visual cortex, at area V1, gets a "virtual image" of the real image appearing in the scene as "seen" by the living beings.

i. No "biological" software appears at the visual cortex. Any type of information is processed by hardware interconnections among different neurons.

j. Any biological information processing is performed by non linear effects. 
k. Although different types of information processing appear in the retina and visual cortex this processing is the result of interchange of information among neurons in the same level. No feedback processes appears in the neural network.

1. The number of levels needed to go from receptor neurons in the retina to V1 layer in the cortex is lower than 15 .

\section{SENSING SYSTEMS EMULATING THE MAMMALIAM VISUAL CORTEX}

Previous considerations allow us to assert some proposals in order to develop sensing systems with the example of sense organs in living bodies.

The first one should be the parallel processing. The advantage of this method may easily be seen with an example. Any animal visual system is able to process the information impinging onto its eyes much faster than with any artificial system. This is due to the fact that the whole information is carried out to the cortex in a parallel way with almost a different channel for each different data.

The second one concerns data processing. These data arriving to the cortex have had, previously, a processing step. This operation has been carried out just after the reception level. Moreover, signals are processed according to their spatial and temporal characteristics. Each stream of data carry two types of information: information about the incident signal to a particular sensing unit and information about how this signal is related with other signals arriving to nearby sensing element. A route may channel data from different situations: because its main sensing element has some signal impinging onto it and there are no signals into adjacent elements, because it has no signal but adjacent elements have or because all of them have signals but with different parameters. Each one of these situations will give rise to a different signal to the cortex. Relations among neurons have some similarities with Boolean functions.

The third one is related with the lower level processing unit, the cortex. Each one of the above mentioned channels have a specific place to arrive. The information carried out to the central processing unit depends on the excited region. Now, this unit process that information according to previous experiences. New signal plus memory dictate the final response. In some cases, signal, plus memory, plus information coming from other adjacent cannels are the origin of subjective interpretation of an event.

In every one of previous considerations, main signals are always of digital-like type. Information about intensity of the stimulus is carried by a burst of pulses, with frequency depending on the signal strength. Analogue to digital conversion takes place at the first processing levels.

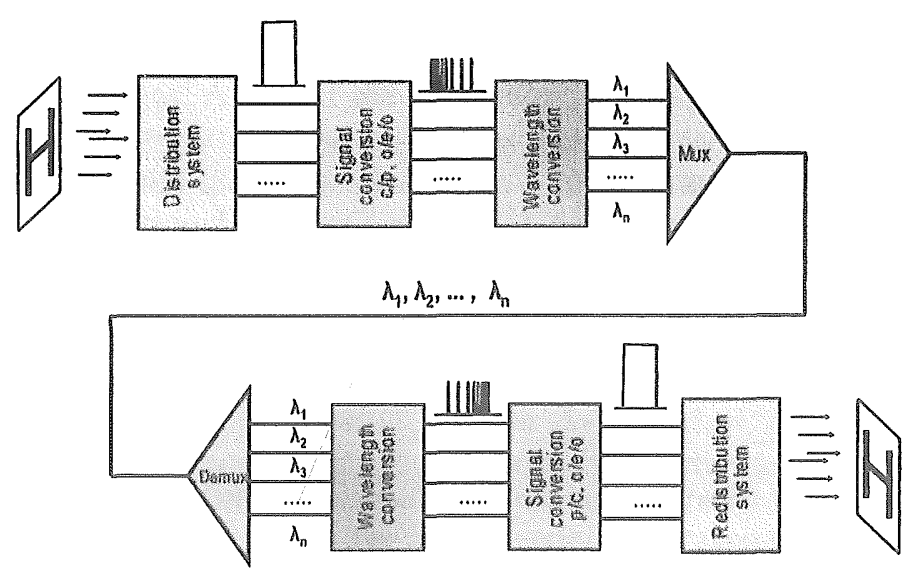

Fig. 8.- General diagram of the WDM image transmission system with biological concepts.

\subsection{Examples of previous considerations}

As a first example of the possible applications in sensing technology, this paper reports now a simple structure able to transfer information in a parallel way. The general structure to be employed is the one shown in Fig. 7. The main objective is to transfer a particular image - an "H" in this figure - to a receptor. The structure is composed by different blocks, each one with a different function. The principal aspect is the way to implement the parallel transmission. A solution is assigning to each one of the neural parallel paths a different wavelength. In this way, different neuronal paths may go through the same physical line and each one of the signals may be processed in an individual basis. This WDM method allows handling as many different signals as optical frequencies are available. Each one of these wavelength corresponds, for instance, to each one of the different line orientations or shapes to be analyzed. Some more details may be seen in ${ }^{6}$.

The second application is related with the possibility to implement systems able to detect spatial contrast or direction sensitivity, according to the rules given in Fig. 4. A possible architecture, based on the mammalian retina 


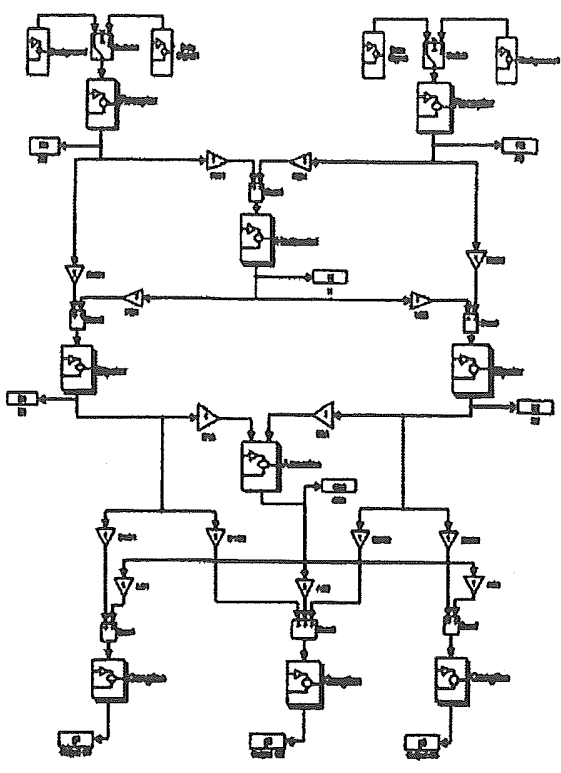

Fig. 9.- Computer simulation of the retina model.

appears in Fig. 8. This structure allows knowing which one of the two receptors get light, the relation between their intensities and if the light goes from left to right or from the right to the left. Building unitary blocks are optical programmable logic cells ${ }^{7-8}$ able to perform as many Boolean functions as neurons do. More details may be seen in ${ }^{9-11}$. Some other systems, based on similar configurations, give the possibility to extract some characteristics of an image as, for instance, its symmetry ${ }^{12}$.

Finally, a last application is related with the possibility to analyze some visual illusions as perceived by living beings in general, and humans in particular. The basis of this new application is the existence of ocular dominance columns appearing in Fig. 7. Influence between them determines a certain uncertainty when two signals go through adjacent paths. This effects, that in humans give rise to illusions, in artificial systems may origin wrong results. Analysis in living beings may help to clarify this problem.

\section{CONCLUSIONS}

This paper has tried to offer a simple presentation of some of the possibilities obtained when ideas coming from the sensorial systems of some animals are employed. Just some concepts have been presented and some working examples obtained from them. The final goal should be opening a door for a new research.

\section{ACKNOWLEDGMENTS}

This work was partly supported by CICYT, grant TIC2003-04309.

\section{REFERENCES}

1. G.M. Shepherd, "The Synaptic Organization of the Brain". Oxford. 1990.

2. "Neuro-vision Systems. Principles and Applications". Eds.: Madam M. Gupta and George K. Knopf. IEEE Press. New York. 1993.

3. J.G. Nicholls, A. Robert M. and B.G. Wallace, "From Neuron to Brain", Sinauer Associates, Inc. Sunderland, MA. 1992.

4. G.M. Shepherd, Neurobiology, New York: Oxford University Press. 1994.

5. J. E. Dowling, The Retina: An Approachable Part of the Brain. Cambridge, MA: The Belknap Press of Harvard University Press, 1987.

6. J.A. Martín-Pereda and A. Gonzalez-Marcos, "Photonic processing subsystem based on visual cortex architecture". Active and Passive Optical Components for WDM Communications III. SPIE, Vol. 5246. Pp. 676 - 685 (2003)

7. A. González-Marcos and J.A. Martín-Pereda, "Digital Chaotic Output from an Optically-Processing Element". Optical Engineering, 35, pp. 525-535 (1996)

8. A. González-Marcos and J.A. Martín-Pereda, "Analysis of irregular behaviour on an optical computing logic cell”. Optics and Laser Technology, 32, 457 - 466 (2000)

9. Jose A. Martin-Pereda and Ana P. Gonzalez-Marcos, "Image characterisation based on the mammalian visual cortex". Applications of digital Image Processing XXII, SPIE, vol. 3808, pp. 614-623, (1999)

10. A. Gonzalez-Marcos and J.A. Martin-Pereda, "Image characterisation based on the human retina and the mammalian visual cortex" 2nd European Medical and Biological Engineering Conference EMBEC'02 Proc. pp. I-862863. (2002).

11. J.A. Martin-Pereda and A. González-Marcos, "A New Approach to Optical Fibre Sensing Techniques based on the Sensory Systems of the Living Bodies". In "Handbook of Optical Fibre Sensing Technology". Ed.: José Miguel LópezHiguera. Wiley, pp. 769-781. 2002.

12. J.A. Martin-Pereda and A. González-Marcos, "A model of the mammalian visual system with Optical Logic Cells". In "Neural Engineering". Ed.: Metin Akay. Wiley. 2004 (in press)

13. J.A. Martin-Pereda and A. Gonzalez-Marcos, "A method to study the perception of subjective image characteristics” 2nd European Medical and Biological Engineering Conference EMBEC'02 Proc. pp. I-864-865. (2002).

14. J.A. Martin-Pereda and A. Gonzalez-Marcos, "Extraction of subjective properties in Image Processing", Photonic Devices and Algorithms for Computing IV. SPIE, vol. 4788-17. pp: 73-82. (2002) 